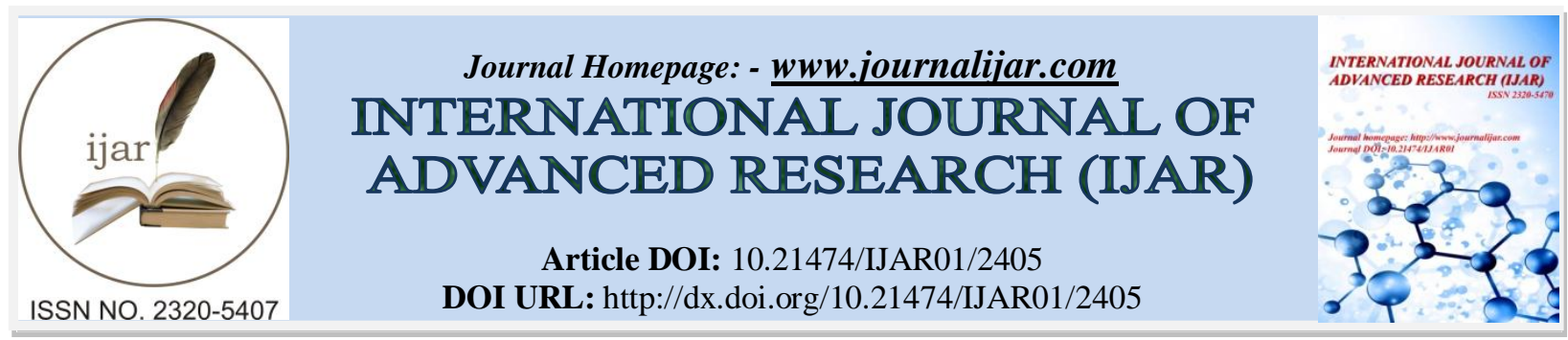

RESEARCH ARTICLE

\title{
JOURNEY TOWARDS THE 3D DENTAL IMAGING- THE MILESTONES IN THE ADVANCEMENT OF DENTAL IMAGING.
}

Dr Kumar Chandan Srivastava, Dr Deepti Shrivastava and Dr Ravi David Austin.

\section{Manuscript Info}

Manuscript History

Received: 19 October 2016

Final Accepted: 20 November 2016

Published: December 2016

Key words:-

Cone beam computed tomography

Conventional computed tomography

Conventional tomography

Digital radiography

Panoramic Radiography

\section{Abstract}

Over the past decades, evolution has been seen in all specialties of dentistry, in terms of unfolding newer facts, treatment approaches and redefining existing concepts. At the back of all these developments, there has been an important role of advancing diagnostic tools, specially imaging methods. Dental imaging has covered a long journey from the simple intra-oral Periapical X-rays to advanced imaging techniques like computed tomography, cone beam computed tomography, magnetic resonance imaging and ultrasonography. Most of these developments started in the medicine, but in no time they had gained a crucial role in diagnosis \& treatment planning for maxillofacial pathologies. Major paradigm shift in imaging was arrived with the conversion of analogue to digital radiography. This has not only made the process simpler and faster but also made image storage, manipulation, retrieval and transmission easier. The threedimensional imaging of complex craniofacial structures is made even more accessible with the advent of cone beam computed tomography. Reduced scan time, radiation dose and enhanced image characteristics are the most promising features of this technology. This paper is to review current advances in imaging technology and their uses in different specialties of dentistry.

Copy Right, IJAR, 2016,. All rights reserved.

\section{Introduction:-}

A Germen physicist, Wilhelm Conrad Rontgen (1845-1923) becomes the first person to observe X-rays, a significant scientific advancement that would ultimately benefit a variety of fields, most of all medicine; by making the invisible visible. On 8 November 1895, Rontgen's discovery occurred accidentally in his Wurzburg, Germany, lab, where he was testing whether cathode rays could pass through glass when he noticed a glow coming from a nearby chemically coated screen. He appellate the rays that caused this glow X-rays because of their unknown nature. Through his series of experiments he learned that X-rays can penetrate human flesh but not higher density substances such as bone or lead and so they can be photographed. Rontgen's discovery was labeled a medical miracle and X-rays soon became an important diagnostic tool in medicine, allowing doctors to see inside the human body for the first time without surgery.

In January 1896, a dentist in Braunschweig, Germany made the first dental radiograph. Using a glass photographic plate wrapped in black rubber dam and with an exposure of 25 minutes, Dr. Otto Walkoff produced this intraoral 
radiograph. Since then, dental radiography has seen oceanic advancement in its technique \& technology and its applications in various fields of dentistry. Broadly, imaging sciences used in dentistry has seen four major breakthroughs till now.

\section{Conventional / Film Based Radiography:-}

In its first phase, intraoral - film based - two dimensional radiography prevailed; with intraoral periapical, bitewing \& occlusal view X-rays. Diagnostic intraoral Periapical radiography has evolved as an inseparable branch of dentistry. Intra oral Periapical radiograph (IOPA) is an important diagnostic aid and routinely used for investigating the Periapical and Periodontal diseases. Proper intra oral Periapical technique not only helps to confirm the diagnosis but also aids treatment planning and management and baseline for assessing the outcome of each pulpal, Periapical, periodontal and other osseous pathologies. Bitewing radiograph outstandingly valuable for detecting inter-proximal caries during its early stages of development, notify secondary caries below the restorations and evaluating the crestal bone height. Among various indications of occlusal radiograph, it remains first choice of imaging in conditions like localization of supernumerary/ impacted teeth and foreign bodies in the jaws and stones in the ducts of sub-mandibular glands. Though it remains a mainstay even in today's dentistry, the major drawbacks were superimposition of neighboring anatomical structures, radiation exposure, compromised image characteristics and depiction of 2 dimensions of a 3 dimensional structure. ${ }^{1}$

\section{Conventional Tomography:-}

The Concept of Tomography marked the beginning of second phase of improvement in imaging world. Film based Conventional tomogram and panoramic radiographs grabbed the attention. Tomography is a procedure used for visualizing selected planes within the patient examined. A single plane is selected and kept in focus, as structures above and below are blurred and out of focus. This is done by moving the $\mathrm{x}$-ray tube in one direction over the patient. The tube and film are linked and synchronized to move in opposite directions. ${ }^{2}$ The procedures and equipment for obtaining a panoramic radiograph have steadily improved since the first one taken in 1934. Present day panoramic machine have multiple moving rotation centers with narrow beam and capturing image in continuous imaging technique in contrast with split images in the past. Panoramic imaging still remains the first choice of investigation when overall assessment of maxillofacial region in respect to dentition, bone support, identification of multiple impacted teeth is required. Its other common indication includes assessment of osseous status of the temporo-mandibular joints and diagnosis of maxillary and mandibular fractures. It made possible to get a sectional image, but radiation exposure, compromised image characteristics and depiction of 2 dimensions of a 3 dimensional structure still remained as a challenge. ${ }^{3}$

\section{Digital Radiography:-}

Third phase came about with the development and widespread use of computer technology with its applications in radiography too. Digital radiography was introduced in the mid-1980s and, with a steady gain in popularity; it is now competing with conventional film based radiography in all radiographic applications. Digital imaging attribute to a method of capturing a radiographic image using a solid-state detectors (sensor) made of silicon that are arranged in an array / matrix of x-ray or light sensitive pixels, converting analog signal into digital via analog to digital convertor (ADC) and processing, presenting and storing the image using a computer. Presently three types of digital radiography systems available for use in dental imaging, namely, CCD-Charge-Coupled Device (direct digital radiography-DDR); CMOS-Complementary Metal Oxide Semiconductor (direct digital radiography); and PSPphoto-stimulable phosphor (indirect digital radiography). All three of these sensors use similar technology with differences in power requirements, internal components, charge transfer and manufacturability. Digital radiography, also known as filmless imaging, has overwhelmed conventional radiography due to its various advantages like reusable image receptors (sensors), considerable reduced image processing time, eliminating use of hazardous materials for processing, added advantage of various image enhancement and analysis tools and ease in long term storage, retrieval and transmission (Teleradiography). ${ }^{4}$ The most talked about positive features of digital radiography is the radiation dose reduction up to $80 \%$, when compared with conventional plain film radiography. It is estimated that the dose reduction for intraoral digital imaging is in the range of $50 \%-60 \%{ }^{2}$ when compared to Espeed film and for extra oral digital imaging, 50\%-70\% ${ }^{5}$ when compared to film-screen combinations. The concept and technology of the digital radiography has been hence extended to all existing intra and extra oral radiographic techniques. 


\section{Computed tomography (CT):-}

Computed tomography (CT) originally known as computed axial tomography (CAT or CT scan) and body section roentgenography, is a medical imaging method that uses computer-processed X-rays to produce tomographic images (virtual 'slices') of specific areas of a scanned object. Unlike the conventional tomography where the image of thin sections is created by blurring out the information from the unwanted regions, the CT image is constructed mathematically using data arising from the section of interest. CT combines thin section imaging of tomography with electronic image acquisition and computerized image construction. ${ }^{6}$ Computed Tomography has its roots in the early part of this century, in 1917, where in the Austrian mathematician; Johann Radon (1887-1956) described a mathematically rigorous inversion formula for reconstruction of an object from its projections. With tremendous research \& experimentation by various scholars over a long period of time, finally the first commercial CT scanner was developed in 1972 by Sir Godfrey N. Hounsfield, an engineer at Electric and Musical Industries (EMI, Limited, a British-based international group of companies) Great Britain.

Numerous innovations have occurred since the first CT scanner was introduced. Scanners can accommodate the entire body, and scans are taken in seconds instead of minutes with increase in number of detectors. These advancements are referred as generations of CTs. There have been four recognized generations of CT scanners and two innovative modifications that vie for the designation of fifth generation.

The first-generation CT scanner was the Mark I (EMI, London, England), the scanner placed at the Atkinson Morley Hospital. In first-generation CT equipment, used single detector element to capture image, opposing to the rectilinear pencil shaped X -ray beam. The method of data collection is based on a rotate/translate principle, in which the beam - detector assembly translate and rotate in $1^{\circ}$ increments for $180^{\circ}$ around the patient's head. To produce one complete scan of the object requires approximately 4.5 to 5.5 minutes. The obvious limitations of firstgeneration CT equipment are lengthy scan time and restricted imaging area.

A $10^{\circ}$ fan beam along with an array of 8 to 30 detectors marked as second-generation scanners. Like firstgeneration scanners, they are based on the rotate/translate principle. The larger beam and increased number of detectors managed to reduce the scan time with improved image resolution improved.

The third-generation or rotate-rotate CT scanner eliminated the need to translate the radiation source and the time lost in having the source achieve its appropriate rotational speed. A larger fan-shaped beam with 288 detectors in the initial model was introduced. Later it was increased to more than 700 detectors. The detectors were arranged in a curvilinear fashion to diminish geometric distortion. As many as 1000 projections could be captured in approximately 1 second. Problem associated with the third-generation scanners was a particular type of artifact created by a single malfunctioning detector. The artifact appeared as a circle in the middle of the slice.

The fourth-generation or rotate-fixed CT scanner uses a continuous circular array and consequently requires no movement of the detector array. More than 2000 detectors are used in some scanners. The speed of the scanner also requires the x-ray tube to be on continuously. X-ray tubes with higher heat capacity have been developed, however, to address the increased workload. The principal advantage of the fourth-generation scanners is that each detector is activated less frequently than the third-generation detectors, and more time is allowed to access the information in each detector and prepare it for the next scan. Scatter and increased patient dose are the major disadvantages of fourth-generation scanners. The problem of scatter has been addressed with additional collimation of the beam and shielding of the detectors. A typical CT slice can be acquired in 1 second. ${ }^{2}$

Newer scanner configurations use multiple $\mathrm{x}$-ray sources or traverse the patient in a novel way in an attempt to capture faster scan times. Spiral CT uses continuous patient and x-ray source motion to decrease the scan times. A new term associated with spiral CT is pitch. Pitch describes the relationship of the speed of patient movement with the rotation of the x-ray source; where higher the pitch, the faster the scan. Higher pitch values however, produce images with missing information. The missing data are calculated or interpolated through software calculations and are subject to false-positive or false-negative findings. ${ }^{8}$

Visualization of multiple, non-superimposed, cross sectional images of both hard and soft tissues of the maxillofacial region was first made possible with CT technology. Benefits of CT were mostly utilized for pre and post surgical assessment of injuries in the maxillofacial skeleton region, especially in detecting complex facial fractures, like those involving the frontal sinus, naso-ethmoidal region and the orbits. ${ }^{9} \mathrm{CT}$ also played a decisive 

role in delimiting surgical margin during the surgical removal of various cyst and tumors in the maxillofacial region.

Cone beam Computed tomography:-

Although with the conventional computed tomography it became possible to have three-dimensional (3D) radiographic imaging, but its application in dentistry has been limited because of cost, access, and dose considerations. These shortcomings finally paved the way for the fourth phase in the advancement of imaging sciences, with the introduction of cone-beam computed tomography (CBCT). The imaging source-detector and the method of data acquisition distinguish cone beam tomography from traditional CT imaging. Traditional CT uses a high-output rotating anode X-ray tube, while cone beam tomography utilizes a low-power, medical fluoroscopy tube that provides continuous imaging throughout the scan. In traditional computerized tomography, an image detector which is arranged in an arc around the patient, records data with a fan-shaped X-ray beam onto producing a single slice image per scan. Each slice must overlap slightly in order to properly reconstruct the images. On the other side, the cone beam technology uses a cone-shaped X-ray beam that transmits onto a solid-state area sensor for image capture, producing the complete volume image in a single rotation. The sensor contains an image intensifier and a CCD camera, or an amorphous silicon flat panel detector. ${ }^{11}$

Widespread acceptance and usage of this technology in various aspects of diagnostic and clinical dentistry is seen due to its various associated benefits. Dimensions \& cost of the equipment makes it very much affordable for the regular use even in a dental office. The single-turn motion image-capture used in cone beam tomography makes the scan time shorter, comparable to panoramic radiography. This reduces the chances of artifacts due to subject movement during the san. However the image processing time varies from 1 minute to 20 minutes, depending upon various factors like selection of 'Field of View' (FOV) and resolution reconstruction algorithm. ${ }^{1}$ Radiation dose is a major competitive factor when compared with conventional CT. This is achieved by choosing the appropriate FOV, tailored according to the patient's need. This enables the collimation of the CBCT primary X-ray beam. As per the Published reports, radiation dose varies from 29 to $477 \mathrm{mSv}$, depending on the type and model of CBCT equipment and FOV selected. Comparing these doses with multiples of a single panoramic dose or background equivalent radiation dose, CBCT provides an equivalent patient radiation dose of 5 to 74 times that of a single film-based panoramic X-ray or 3 to 48 days of background radiation. When compared with conventional CT for maxillofacial imaging (approximately $2000 \mathrm{mSv}$ ), CBCT provides substantial dose reductions of between $98.5 \%$ and $76.2 \%$. CBCT demonstrate images in three orthogonal planes (axial, sagittal, and coronal). Basic enhancements include zoom or magnification, window/level, and the ability to add annotation. Cursor-driven measurement algorithms provide the clinician with an interactive capability for real-time dimensional assessment. Onscreen measurements provide dimensions free from distortion and magnification. Because of the isotropic nature of the volumetric data sets, they can be sectioned nonorthogonally. Most software provides for various nonaxial 2D images, referred to as multi planner reconstruction (MPR). Such MPR modes include oblique, curved planar reformation (providing "simulated" distortion-free panoramic images), and serial transplanar reformation (providing cross-sections), all of which can be used to highlight specific anatomic regions and diagnostic tasks which is important, given the complex structure of the maxillofacial region. CBCT produce isotropic volumetric image, which means the voxels generated have equal dimension in all three planes. This property contributes to its high resolution, accuracy, and reproducibility of $\mathrm{CBCT}$ images. ${ }^{11}$

Despite its wide application, it also bears some Limitations related to the "cone-beam" projection geometry, detector sensitivity, and contrast resolution that produces images that lack the clarity and usefulness of conventional CT images. The clarity of CBCT images is affected by artifacts, noise and poor soft tissue contrast. ${ }^{12}$

Applications of CBCT in Various branches of dentistry:-

Conventional film based radiography remains mainstay in diagnosis \& treatment planning in the practice of endodontic. For situations where information in 3 dimensions is required like in vertical root fractures, failure of endodontic treatment for unknown reasons, a mandate input from CBCT images can be expected. They provide crucial information in all phases of treatment namely pre operative assessment, intraoperative and post operative evaluation. During pre operative phase, it helps in correct assessment of periapical lesions ${ }^{13}$, differentiation between periapical cyst and granuloma ${ }^{14}$, assessment of internal architecture of tooth including size of pulp chamber; number, length, curvature and calcification in root canal ${ }^{15}$, trabecular pattern and morphology and condition of roots. It will also help to reveal dentoalveolar trauma, and finally help in pre-surgical planning. During intraoperative phase, CBCT will provide is a precise measurement of canals which in turn improves prognosis of 
biomechanical preparation and obturation. In Post operative phase, Imaging is important in evaluating the results of previous therapy, delayed healing, evaluating potential obstacles to retreatment, as well as surgical considerations. ${ }^{16}$ CBCT has an extensive application in the field of implant dentistry. It plays a significant role in pre, intra and post operative phase. In first phase it helps in estimation of quality and quantity of available bone; give precise measurement about the proximity of vital structure from the proposed site of implant placement. ${ }^{17}$ With combination of CBCT and implant surgical softwares, virtual surgeries can be performed, which enhance the success rate of implant surgery. ${ }^{18}$ Post operatively CBCT images will help in judgment of healing and osseointegration. ${ }^{19}$

CBCT has justified its use in the detection and evaluation of intrabony defects and furcation involvements, dehiscence, fenestration and periodontal cysts. ${ }^{20}$ It aids in the treatment planning by choosing absorbable membrane with the optimal shape to fit into interproximal bony defects, and thus shorten the time required for the guided tissue regeneration. It has also proved its utility in evaluation of outcome in case of regenerative surgeries. ${ }^{21}$

The applications of CBCT in orthodontics are numerous but its use on routine basis is still questionable as it deals with young patients who will be getting more radiation exposure with this imaging modality. It is taken for the purpose of assessment of skeletal growth pattern, severity of tooth impaction, and upper airway evaluation for possible obstructions. It can influence treatment planning of orthodontic cases who need buccal tooth movement and arch expansion. ${ }^{22,23}$

Although Conventional CT is used routinely in the diagnosis of maxillofacial pathology, but with the given higher resolution, lower radiation dose, and lower cost of CBCT in imaging the maxillofacial region, it stands to reason that CBCT can easily replace conventional CT in this regard. Three dimensional imaging of cysts and tumors of the maxillofacial region can give the surgeon the vital information necessary for planning surgical execution; ${ }^{24}$ with volumetric analysis, this can help anticipate the need for and volume of a potential graft for reconstruction. It also gives vital inputs in case of mid face fracture, orbital fracture and even in impaction surgery. ${ }^{25,}{ }^{26}$ Published data states that if CBCT images are is used in pre operative planning, it would help surgeons to choose the most conservative surgical approach which in turn will reduce the mortality and morbidity. ${ }^{27}$

\section{Conclusion:-}

The field of dental imaging has witnessed extensive advancement in its technology. The root cause of which is the constant efforts by the research scholars to overcome the limitations in the existing technology. Today with the advent of CBCT have undoubtedly revolutionized dental diagnostics and treatment planning. This technology is capable of providing accurate, submillimeter-resolution images of desired specific area (via selection of FOV) in formats allowing 3D visualization of the complexity of the maxillofacial region within the premises of dental office. Constant efforts are been made in refining CBCT machine with even lesser scan time, improving image fidelity, including soft tissue contrast. The increasing availability of this technology provides the practitioner with a modality that can help to detect pathologies in very early stages, which ultimately will help to reduce morbidity and mortality and improve the quality of life of the patients.

\section{References:-}

1. White SC, Pharoah MJ. Oral Radiology: Principles and Interpretation. 5th Ed. St. Louis: Mosby Inc., 2004.

2. Whaites E. Essentials of dental radiography and radiology. 3rd Ed. London, Churchill Livingstone 2002.

3. Farman AG, Farman TT. Extraoral and panoramic systems. Dent Clin North Am. 2000; 44: 257-72.

4. Langland OE, Langlais RP, Preece JW. Principles of dental imaging. 2nd ed. Philadelphia: Lippincott Williams \& Wilkins, 2002.

5. Visser H, Rödig T, Hermann KP. Dose reduction by direct digital cephalometric radiography. Angle Orthod 2001; 71: 159-163.

6. Brooks SL. Computed tomography. Dent Clin North Am 1993; 37: 575- 90.

7. Goldman LW. Principles of CT and CT technology. J Nucl Med Technol 2007; 35: 115-128.

8. Scarfe WC. Imaging of maxillofacial trauma: evolutions and emerging revolutions. Oral Surg Oral Med Oral Pathol Oral Radiol Endod 2005; 100: S75-S96.

9. Davidson MJ, Daly BD, Russell JL. The use of computed tomography in the management of facial trauma by British oral and maxillofacial surgeons. Br J Oral Maxillofac Surg 1991; 29: 80-1.

10. Abrahams JJ. Dental CT imaging: a look at the jaw. Radiology 2001; 219: 334-45. 
11. Scarfe WC, Farman AG. What is cone beam CT and how does it work? Dent Clin N Am 2008; 52: 707-30.

12. Katsumata A, Hirukawa A, Noujeim M, Okumura S, Naitoh M, Fujishita M, Ariji E, Langlais RP. Image artifact in dental cone-beam CT. Oral Surg Oral Med Oral Pathol Oral Radiol Endod 2006; 10: 652- 57.

13. Estrela C, Bueno MR, Leles CR, Azevedo B, Azevedo JR. Accuracy of cone beam computed tomography and panoramic and periapical radiography for detection of apical periodontitis. J Endod 2008; 34: 273-79.

14. Lofthag-Hansen S, Huumonen S, Gröndahl K, Gröndahl HG. Limited cone-beam CT and intraoral radiography for the diagnosis of periapical pathology. Oral Surg Oral Med Oral Pathol Oral Radiol Endod 2007; 103: 11419.

15. Patel S, Dawood A, Ford TP, Whaites E. The potential applications of cone beam computed tomography in the management of endodontic problems. Int Endod J 2007; 40: 818-30.

16. Scarfe WC, Levin MD, Gane D, Farman G. Use of Cone Beam Computerized Tomography in Endodontics. Int J Dent 2009: 1-20.

17. Hatcher DC, Dial C, Mayorga C. Cone beam CT for presurgical assessment of implant sites. J Calif Dent Assoc 2003; 31: 825-33.

18. Chan HL, Misch K, Wang HL. Dental imaging in implant treatment planning. Implant Dent 2010;19: 288-98.

19. Worthington P, Rubenstein J, Hatcher DC. The role of cone-beam computed tomography in the planning and placement of implants. J Am Dent Assoc 2010; 141: 19S-24S.

20. Kasaj A, Willershausen B. Digital volume tomography for diagnostics in periodontology. Int J Comput Dent 2007; 10: 155-68.

21. Ito K, Yoshinuma N, Goke E, Arai Y, Shinoda K. Clinical application of a new compact computed tomography system for evaluating the outcome of regenerative therapy: a case report. J Periodontol 2001; 72: 696-702.

22. Nurko C. Three-dimensional imaging cone bean computer tomography technology: an update and case report of an impacted incisor in a mixed dentition patient. Pediatr Dent 2010; 32: 356-60.

23. Kumar V, Ludlow J, Soares Cevidanes LH, Mol A. In Vivo Comparison of Conventional and Cone Beam CT Synthesized Cephalograms. Angle Orthod 2008; 78: 873-79.

24. Marques YM, Botelho TD, Xavier FC, Rangel AL, Rege IC, et al. Importance of cone beam computed tomography for diagnosis of calcifying cystic odontogenic tumour associated to odontoma. Report of a case. Med Oral Patol Oral Cir Bucal 2010; 15: e490-93.

25. Blessmann M, Pohlenz P, Blake FA, Lenard M, Schmelzle R, Heiland M. Validation of a new training tool for ultrasound as a diagnostic modality in suspected midfacial fractures. Int J Oral Maxillofac Surg 2007; 36: 50106.

26. Zizelmann C, Gellrich NC, Metzger MC, Schoen R, Schmelzeisen R, Schramm A. Computer-assisted reconstruction of orbital floor based on cone beam tomography. Br J Oral Maxillofac Surg 2007; 45: 79-80.

27. Bortoluzzi MC, Manfro R. Treatment for ectopic third molar in the subcondylar region planned with cone beam computed tomography: a case report. J Oral Maxillofac Surg 2010; 68: 870-72. 